\title{
Vector Potential Equivalent Circuit Based on PEEC Inversion
}

\author{
Hao Yu, Student Member, IEEE, and Lei He, Member, IEEE
}

\begin{abstract}
The geometry-integration based vector potential equivalent circuit (VPEC) was introduced to obtain a localized circuit model for inductive interconnects in [1]. In this paper, we show that the method in [1] is accurate only for the two-body problem. We derive N-body VPEC models based on geometry integration and inversion of inductance matrix under the PEEC model, respectively. Both VPEC models are derived from first principles and are accurate compared to the full PEEC model. The resulting circuit matrix $\hat{G}$ can be analyzed directly by existing simulation tools such as SPICE, and the simulation time of VPEC model is $47 X$ smaller than that for PEEC model for a bus structure with 256 wire segments. It is also passive and strictly diagonal dominant, which leads to efficient circuit sparsification methods such as numerical and geometry based sparsifications. Compared to the full PEEC model, the sparsified VPEC models are orders of magnitude faster and produce waveforms with very small error.
\end{abstract}

\section{INTRODUCTION}

As VLSI technology advances with decreasing feature size as well as increasing operating speed and global interconnect length, an increasing portion of interconnects should be modeled as RLC circuits [2]. Although these interconnects can be accurately modeled by Partial Element Equivalent Circuit (PEEC) [3], the resulting full PEEC circuit may have an extremely high complexity for circuit analysis. Because the partial inductance matrix in PEEC is not diagonal dominant, simply truncating off-diagonal elements leads to negative eigenvalues such that the truncated matrix loses the property of passivity [4]. Several inductance sparsification methods have been proposed with guaranteed passivity. The return-loop inductance model [5] assumes that the current for a signal wire returns from the nearest ground wires sandwiching the signal wire. It loses accuracy by ignoring coupling between signal wires not in the same "halo". The shift-truncation model [6] directly calculates a sparse inductance matrix by assuming that the current returns from a shell with radius $r_{0}$. However, it is difficult to define $r_{0}$ to obtain the desired accuracy. The inverse-truncation model [7] replaces the inductance matrix by its inversion, called $K$ matrix or susceptance. $K$ matrix is diagonal dominant and small-valued off-diagonal elements can be truncated without affecting the passivity. Because $K$ is a new circuit element that is not considered in conventional circuit analysis such as SPICE, new circuit analysis tools need to be developed [8]. Further, inversion of truncated $K$ matrix

This research was partially supported by the NSF CAREER Award 0093273, and grants from Intel and Analog Devices.

H. Yu is with the Electrical Engineering Department, University of California at Los Angeles, Los Angeles, CA 90095 USA (email:hy255@ee.ucla.edu).

L. He is with the Electrical Engineering Department, University of California at Los Angeles, Los Angeles, CA 90095 USA (email:1he@ee.ucla.edu). is proposed to avoid using $K$ in simulation [9], and wire duplication is used to construct a complexity-reduced circuit that is equivalent to the circuit under the inductance matrix or under the truncated $K$ matrix [10].

Using equivalent magnetic resistance to model inductive interconnects, the geometry-integration based vector potential equivalent circuit (VPEC) is introduced in [1]. The resulting circuit model can be analyzed by SPICE, and shows a good potential for circuit sparsification. This paper presents an indepth study on VPEC. In Section 2, we show that the VPEC method in [1] is accurate only for the two-body problem, and derive an accurate N-body VPEC models based on geometry integration. In Section 3, we introduce a new N-body VPEC model using inversion of inductance matrix under the PEEC model. Both VPEC models are derived from first principles and are accurate compared to the full PEEC model. The integration based VPEC model needs a FastHenry [11]-like three-dimensional field solver developed from scratch, but the inversion based VPEC model can be easily obtained using the partial inductance matrix generated by FastHenry. Further, we prove that the circuit matrix $\hat{G}$ resulting from the VPEC model is passive and strictly diagonal dominant. As a by-product, the $\hat{G}$ matrix can be used to justify from first principles the $K$ matrix (or susceptance) based sparsification methods. In Section 4, we present efficient circuit sparsification methods leveraging the passivity of $\hat{G}$ matrix. We conclude the paper in Section 5.

\section{INTEGRATION BASED VPEC}

In this section, we first use the two-body problem to illustrate the concept of VPEC model, then extend VPEC to the N-body problem.

\section{A. Two-Body Problem}

Same as in FastHenry [11], the long and thin conductor in integrated circuits can be divided into a number of rectilinear filaments. Given the magneto-quasi-static assumption, the current is constant in the current direction assumed as $z$-axis in this paper, and it is uniform over the cross-section of the current flow (i.e., uniform over the cross-section of filament). For VPEC, the region of filament is extended to include the space between two adjacent filaments as shown in Fig. 1, such that the two extended regions touch each other. To be precise, we call the extended filament as hyper-filament (in short, $h$ filament). If the original filaments already touch each other, the h-filaments are equivalent to the filaments. In this paper, we use the superscripts $x, y, z$ to denote spacial components of a vector variable. Let $\mathbf{A}$ be the vector potential, then $A^{z}$ 


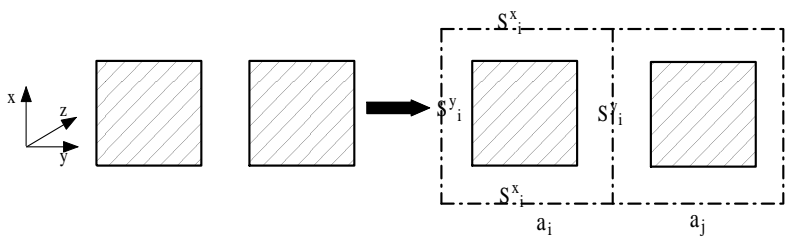

(a)

(b)

Fig. 1. Expansion of two filaments in (a) to hyper-filaments in (b). The surface $S_{i}^{y}$ locates in the middle of two h-filaments.

is its $z$-direction component. We use the subscripts $i$ and $j$ for variables associated with h-filaments $a_{i}$ and $a_{j}$. Without loss of generality, two h-filaments with cross-section in $x-y$ plane and an identical length $l$ in $z$-direction are studied in the two body problem. We start with the differential Maxwell equations in the formalism of $A^{z}$ :

$$
\begin{gathered}
\nabla^{2} A^{z}=-\mu J^{z} \\
\frac{\partial A^{z}}{\partial t}=-E^{z}-\nabla^{z} \phi
\end{gathered}
$$

where the vector potential $\mathbf{A}$ is in $z$-direction same as current density $\mathbf{J}, \mathbf{E}$ is electrical field, and $\phi$ is the scalar potential. Because $J^{z}=J_{i}^{z}+J_{j}^{z}$, the total vector potential is $A^{z}=$ $A_{i}^{z}+A_{j}^{z}$, where $A_{i}^{z}$ is determined by $J_{i}^{z}$ of h-filament $a_{i}$ :

$$
A_{i}^{z}=\frac{\mu}{4 \pi} \int d \mathbf{r}_{i}^{\prime} \frac{J_{i}^{z}}{\left|\mathbf{r}-\mathbf{r}_{i}^{\prime}\right|}
$$

where $\left|\mathbf{r}-\mathbf{r}_{i}^{\prime}\right|$ is the distance between the source and destination points. $A_{j}^{z}$ of h-filament $a_{j}$ can be obtained similarly. Furthermore if (1) is integrated within the volume $\Omega_{i}$ of hfilament $a_{i}$, using Gauss' law:

$$
\int_{S} d \mathbf{S} \cdot \mathbf{a}=\int_{\Omega} d \Omega \nabla \cdot \mathbf{a},
$$

we can obtain the following integral equation:

$$
\int_{S_{i}} d \mathbf{S} \cdot \nabla A^{z}+\mu \int_{\Omega_{i}} d \Omega J_{i}^{z}=0
$$

where $S_{i}$ is the surface of h-filament $a_{i}$, including $S_{i}^{x}$ and $S_{i}^{y}$ (see Fig. 1), and only the contribution of $J_{i}^{z}$ is counted because the integration is inside $a_{i}$. An effective resistance (called equivalent magnetic resistance, in short, EMR) is defined as

$$
\hat{R}_{i j}=\mu \frac{\left(A_{i}^{z}\left|S_{i}-A_{j}^{z}\right|_{S_{i}}\right)}{\left.\int_{S_{i}} d \mathbf{S} \cdot \nabla A^{z}\right|_{S_{i}}}
$$

to model (i.e., replace) the mutual inductive coupling between $a_{i}$ and $a_{j}$. Its value is determined by the average of $A_{i}^{z}$ and $A_{j}^{z}$, both evaluated at surface $S_{i}$. Note that the definition of EMR in this paper is slightly different from [1] but more precise. For the simplicity of presentation, we define:

$$
A_{i}=\left.A_{i}^{z}\right|_{S_{i}}, \quad A_{j}=\left.A_{j}^{z}\right|_{S_{i}}
$$

Note that the gradients of $A_{i}^{z}$ and $A_{j}^{z}$ at surface $S_{i}$ are opposite to each other.

Moreover, there exists a ground EMR taking into account the self inductive effect. The ground EMR of $a_{i}$ is given by:

$$
\hat{R}_{i 0}=\mu \frac{\left.A_{i}^{z}\right|_{S_{i}}}{\left.\int_{S_{i}} d \mathbf{S} \cdot \nabla A_{i}^{z}\right|_{S_{i}}}
$$

Because the current is constant along $z$-direction, the volume integral of current density is reduced to $l I_{i}$, where $I_{i}$ is the electrical current at $a_{i}$. Therefore (5) is simplified as:

$$
\frac{A_{i}}{\hat{R}_{i 0}}+\frac{\left(A_{i}-A_{j}\right)}{\hat{R}_{i j}}=-l I_{i}
$$

A vector potential current source $\hat{I}_{i}$ can be defined as:

$$
\hat{I}_{i}=l I_{i}
$$

which is controlled by the electrical current $I_{i}$.

On the other hand, integrating (2) along $z$-direction at the h-filament surface $S_{i}$ leads the following inductive electropotential drop at $a_{i}$ :

$$
l \frac{\partial A_{i}}{\partial t}=-V_{i}
$$

Consequently the voltage-controlled vector potential voltage source $\hat{V}_{i}$ is defined as:

$$
\hat{V}_{i}=V_{i} / l
$$

The VPEC model for two h-filaments includes following components [1] (see Fig. 2): (i) four nodes $\left(I N_{i}, n_{i 1}, n_{i 2}\right.$, $O U T_{i}$ ) for each h-filament $a_{i}$; (ii) the pre-calculated resistance and capacitance between $I N_{i}$ and $n_{i 1}$; (iii) an electrical current source $I_{i}$ between $n_{i 1}$ and $n_{i 2}$ controlling a vector potential current source $\hat{I}_{i}$ (see (10)); (iv) a vector potential voltage source $\hat{V}_{i}$ controlled by the vector potential current source $\hat{I}_{i} ;(\mathrm{v})$ an electrical voltage source $V_{i}$ between $n_{i 2}$ and $O U T_{i}$ controlled by the vector potential voltage source $\hat{V}_{i}$ (see (12)); (vi) effective resistances including ground $\hat{R}_{i 0}$ (see (8)) and coupling $\hat{R}_{i j}$ (see (6)) resistances to account for the strength of inductances; and (vi) a unit inductance $L_{i}$ to account for time derivative of the electrical current source $I_{i}$. It can be easily extended for the general three dimensional current distribution by adding two more VPEC circuits for $x$ and $y$ components. In essence, the VPEC model uses a resistance network plus unit self inductance and controlled voltage/current sources to replace the mutual inductance network. Although the VPEC model introduces more circuit elements, experiments in Section IV will show that it reduces simulation time for interconnects with nontrivial size. 


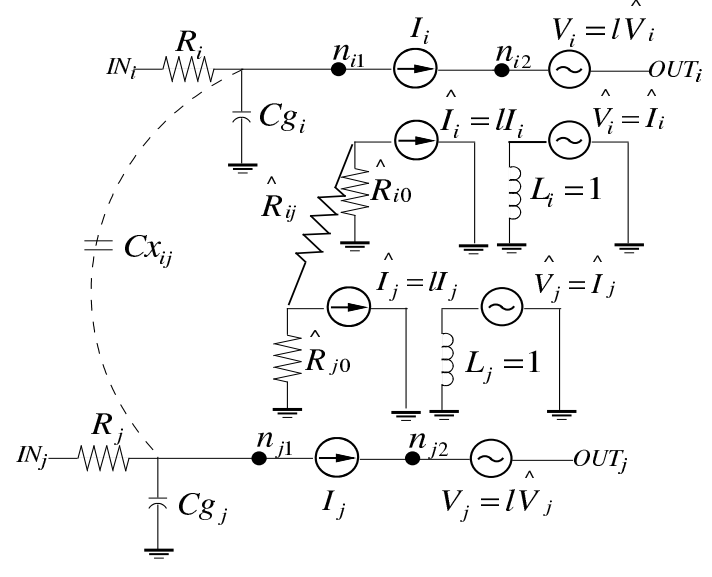

Fig. 2. The Vector Potential Equivalent Circuit model for two h-filaments.

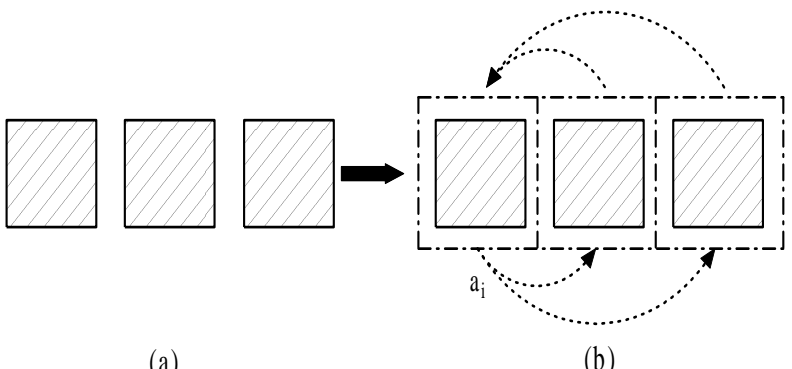

Fig. 3. Expansion of three filaments in (a) to hyper-filaments in (b). The magnetic flux starting from or ending at h-filament $a_{i}$ is not local.

\section{B. N-Body Problem}

We first expand $\mathrm{N}$ filaments into h-filaments as illustrated in Fig. 3, and extend the VPEC model to the N-body problem by collocating all possible coupling pairs independently. Collocation is a common approach to construct the system equations [11], [12]. We collocate the vector potential drops from $a_{i}$ to all the other h-filaments, and obtain the following equation at $a_{i}$ :

$$
\frac{A_{i}}{\hat{R}_{i 0}}+\sum_{j \neq i} \frac{\left(A_{i}-A_{j}\right)}{\hat{R}_{i j}}=-l I_{i}
$$

Note that the above summation is not local. However, in [1] the summation is local, and there are at most six coupling $\hat{R}_{i j}$ for each h-filament in three-dimension. The author obtained the localized model based on the analogy between (5) and the conduction current flow at a surface $S: \mathbf{I}=-\sigma \int_{S} d \mathbf{S} \cdot \nabla \phi$. The later is exactly the Ohm's law, which means the conduction current $\mathbf{I}$ at $S$ is only related to the flux of the electrical field $\mathbf{E}$ $(-\nabla \phi)$ at that surface. In the electro-quasi-static condition, $\mathbf{E}$ is along the same direction of $\mathbf{I}$ because no charge accumulated at the surface. However, for our N-body magneto-quasi-static problem, the flux in (5) is not along the conduction current direction $z$. Therefore it is related not only to the localized $\hat{R}_{i j}(j=i \pm 1)$, but also to all other $\hat{R}_{i j}(j \neq i)$. The experiments in Section 4.1 also show that compared to the full PEEC model, our VPEC model considering all neighbors is accurate, but the localized VPEC model from [1] is not accurate.

Furthermore there is no rigorous methodology to extract the equivalent magnetic resistance in [1]. We propose the following integration based method to obtain the EMRs: (i) calculate the distribution of $\mathbf{A}$ for the given input current distribution by (1) and (3); (ii) evaluate both the average vector potential difference between $A_{i}^{z}$ and $A_{j}^{z}$ and the surface integral by gradient of $A^{z}$ at $S_{i}$ according to (6) and (8). However, it is difficult to determine the appropriate size for each h-filament in numerical integration. In the next section, we propose a new inversion-based VPEC model without using integration.

\section{VPEC VIA PEEC INVERSION}

In this section we first present a closed-form relation between VPEC and the inversion of PEEC, then prove that the new circuit matrix $\hat{G}$ for VPEC model is passive and strictly diagonal dominant.

\section{A. $\hat{G}$ Matrix}

To obtain the circuit equation based on the electrical voltages and currents, we first take the time derivative at both sides of (13) and obtain

$$
\frac{\partial A_{i} / \partial t}{\hat{R}_{i 0}}+\sum_{j \neq i} \frac{\left(\partial A_{i} / \partial t-\partial A_{j} / \partial t\right)}{\hat{R}_{i j}}=-l \frac{\partial I_{i}}{\partial t}
$$

and then use (11) to replace the time derivative of vector potential. Consequently we obtain:

$$
\frac{V_{i}}{\hat{R}_{i 0}}+\sum_{j \neq i} \frac{V_{i}-V_{j}}{\hat{R}_{i j}}=l^{2} \frac{\partial I_{i}}{\partial t}
$$

It leads to

$$
\left(1 / \hat{R}_{i 0}+\sum_{j \neq i} 1 / \hat{R}_{i j}\right) V_{i}+\sum_{j \neq i}\left(-1 / \hat{R}_{i j}\right) V_{j}=l^{2} \frac{\partial I_{i}}{\partial t}
$$

We define the circuits matrix of VPEC model as:

$$
\hat{G}_{i j}=-1 / \hat{R}_{i j}, \quad \hat{G}_{i i}=1 / \hat{R}_{i 0}+\sum_{j \neq i} 1 / \hat{R}_{i j}
$$

The system equations can be written as:

$$
\hat{G}_{i i} V_{i}+\sum_{j \neq i} \hat{G}_{i j} V_{j}=l^{2} \frac{\partial I_{i}}{\partial t}
$$

Compared to the following system equations based on $K$ matrix [8] or the susceptance matrix $S$ in [9]:

$$
K_{i i} V_{i}+\sum_{j \neq i} K_{i j} V_{j}=\frac{\partial I_{i}}{\partial t}
$$




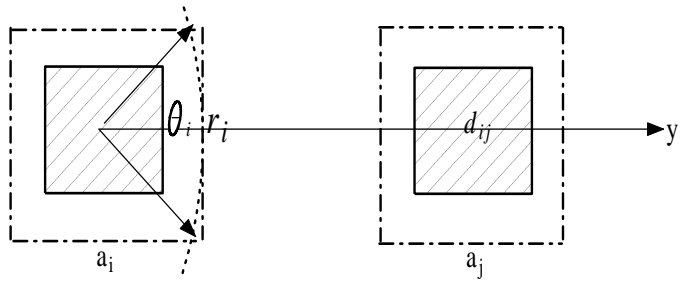

Fig. 4. Directly calculation $\hat{R}_{i j}$ of two long and thin h-filaments. They are not adjacent to each other in general.

where $K=L^{-1}$ and $L$ is the partial inductance matrix, we find that $\hat{G}$ and $K$ only differ by a factor of $l^{2}$, i.e.

$$
l^{2} K_{i j}=\hat{G}_{i j}, \quad l^{2} K_{i i}=\hat{G}_{i i}
$$

Therefore starting with the $L$ matrix under PEEC model, we can first obtain $\hat{G}$ matrix via (20), and then derive $\hat{R}$ matrix via (17). Because the major computation step is inversion of $L$ matrix, we call this method as inversion based VPEC model. Furthermore, (20) can be viewed as how to derive the $K$ matrix based model in [7] from first principles.

\section{B. Property}

Lemma 1: Let $I_{i}$ and $I_{j}$ be currents for two h-filaments $a_{i}$ and $a_{j}$, and $l_{i}$ and $l_{j}$ be lengths for $a_{i}$ and $a_{j}$. If $I_{i} l_{i}=I_{j} l_{j}=$ $I l$, then magnetic resistance $\hat{R}_{i j}, \hat{R}_{i 0}$ and $\hat{R}_{j 0}$ are all positive. Proof: We assume that the centers of $a_{i}$ and $a_{j}$ are $(0,0)$ and $\left(0, d_{i j}\right)$ (see Fig. 4). The vector potentials of h-filaments $a_{i}$ and $a_{j}$ are given:

$$
A_{i}^{z}=-\frac{\mu I l}{2 \pi} \ln \left(r / r_{0}\right), \quad A_{j}^{z}=-\frac{\mu I l}{2 \pi} \ln \left(\left|r-d_{i j}\right| / r_{0}\right)
$$

where $\left|r-d_{i j}\right|=\sqrt{\left(x-d_{i j}\right)^{2}+y^{2}}$, and $r_{0}$ is the dimension of filament. We define $\alpha_{i j}=\frac{\left|r_{i}-d_{i j}\right|}{r_{i}}$ describing the distance difference between $a_{i}$ and $a_{j}$. Note that $\alpha_{i j} \geq 1$ as $d_{i j} \geq 2 r_{i}$ according to the definition of h-filament.

The vector potential difference between $A_{i}^{z}$ and $A_{j}^{z}$ at surface $S_{i}$ is:

$$
\left.\left(A_{i}^{z}-A_{j}^{z}\right)\right|_{S_{i}}=\frac{\mu I l}{2 \pi} \ln \left(\alpha_{i j}\right)
$$

where $r_{i}$ is the distance from the origin to the point on the surface $S_{i}$ with coordinate $\left(x_{i}, y_{i}\right)$.

Moreover, the magnetic flux at surface $S_{i}$ is:

$$
\begin{array}{r}
\left.\int_{S_{i}} d \mathbf{S} \cdot \nabla\left(A_{i}^{z}+A_{j}^{z}\right)\right|_{S_{i}} \\
=\frac{\mu I l}{2 \pi}\left(l \theta_{i}\right)\left(1-\alpha_{i j}^{-1}\right)
\end{array}
$$

where $\theta_{i}\left(0 \leq \theta_{i} \leq 2 \pi\right)$ is the angle for the surface integration at $S_{i}$ with respect to the center of $a_{i}$. Therefore according to the definition of $R_{i j}$ in (6), we have $\hat{R}_{i j}>0$ for $j \neq i$. Similarly, we can prove $\hat{R}_{i 0}>0$ and $\hat{R}_{j 0}>0$. Both $\hat{R}_{i 0}$ and $\hat{R}_{j 0}$ are ground magnetic resistance for self inductance.
By refining h-filaments and segmenting the $\mathrm{h}$-filament with larger current, we can always achieve $I_{i} l_{i}=I_{j} l_{j}$. For the application in PEEC inductance extraction, it means by segmenting the length of longer h-filaments according to shorter h-filaments, we can always obtain positive EMR elements for VPEC model. Therefore, we have the following Lemma 2:

Lemma 2: All magnetic resistances in VPEC model are positive.

We may prove the following theorem by Lemma 2:

Theorem 1: Circuit matrix $\hat{G}$ in VPEC model is passive ${ }^{1}$ and strictly diagonal dominant.

Proof: According to (17) and Lemma 2, we have

$$
\sum_{j \neq i}\left|\hat{G}_{i j}\right|=\sum_{j \neq i} 1 / \hat{R}_{i j}
$$

Because $R_{i 0}$ is also positive, we have

$$
\sum_{j \neq i}\left|\hat{G}_{i j}\right|<1 / \hat{R}_{i 0}+\sum_{j \neq i}^{N} 1 / \hat{R}_{i j}=\hat{G}_{i i}
$$

or

$$
\hat{G}_{i i}>\sum_{j \neq i}\left|\hat{G}_{i j}\right|
$$

I.e., the circuit matrix $\hat{G}$ is strictly diagonal dominant. Furthermore, it is also is passive according to the Gersgorin Circle Theorem [13].

Note that truncating off-diagonal entries from a strictly diagonal dominant matrix still leads a passive matrix. Intuitively, truncating small off-diagonal entries in $\hat{G}$ matrix (equivalent to truncating larger off-diagonal entries in $\hat{R}$ matrix) results in ignoring larger magnetic resistors in the equivalent magnetic resistances network. Based on Theorem 1, such truncation/sparsification leads to passive circuit models. Furthermore, larger magnetic resistors are less sensitive to and also contribute less to current change. Therefore, such sparsification may have a bounded accuracy loss, as shown by two sparsification procedures in Section 4.

\section{EXPERIMENTAL RESULTS}

We have implemented the inversion-based VPEC method in $\mathrm{C}$ code with the following steps: (i) generate partial inductance matrix $L$ by FastHenry or formula from [14], [15]; (ii) inverse $L$ by LU decomposition; (iii) calculate $\hat{G}$ and then $\hat{R}$; and (iv) generate VPEC model using $\hat{R}$. We assume each wire segment is modeled by one h-filament, and consider coupling between any pair of segments (including segments in a same line) unless specified otherwise.

The following simplified formula is employed for selfinductance of the long and thin h-filament [14]:

$$
L_{i i}=\frac{\mu l_{i}}{2 \pi}\left[\ln \left(\frac{2 l_{i}}{w_{i}+t_{i}}\right)+0.5+0.2235 \frac{w_{i}+t_{i}}{l_{i}}\right]
$$

where $l_{i}, w_{i}$ and $t_{i}$ are the wire length, width and thickness

\footnotetext{
${ }^{1}$ Precisely, the matrix is positive definite, and the resulting circuit model is passive. In short, we say the matrix is passive in this paper.
} 


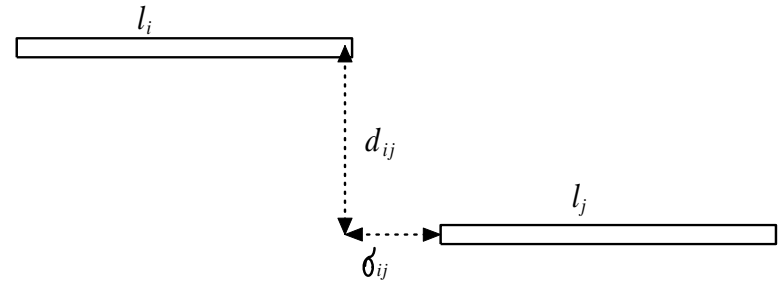

Fig. 5. Two parallel h-filaments with a geometric mean distance $d_{i j}$ apart.

respectively. Furthermore, for the partial mutual inductance between two wires as shown in Fig. 5, the following simplified formula [14] is given:

$$
\begin{aligned}
L_{i j}=\frac{\mu}{4 \pi} & {\left[\alpha \sin ^{-1} \frac{\alpha}{d_{i j}}-\beta \sin ^{-1} \frac{\beta}{d_{i j}}\right.} \\
-\gamma & \sinh ^{-1} \frac{\gamma}{d_{i j}}+\delta_{i j} \sinh ^{-1} \frac{\delta_{i j}}{d_{i j}} \\
& -\sqrt{\alpha^{2}+d_{i j}^{2}}+\sqrt{\beta^{2}+d_{i j}^{2}} \\
+\sqrt{\gamma^{2}+d_{i j}^{2}} & \left.-\sqrt{\delta_{i j}^{2}+d_{i j}^{2}}\right]
\end{aligned}
$$

where $\alpha=l_{i}+l_{j}+\delta_{i j}, \beta=l_{i}+\delta_{i j}$, and $\gamma=l_{j}+\delta_{i j}$. Note that $\delta_{i j}$ becomes negative when the two wires are overlapped. Furthermore, when the condition $l>>d$ holds, there are more efficient approximated formula can be employed as in [15].

We assume copper interconnect and low-k $(\epsilon=2)$ dielectric, and use FastCap to extract capacitance. Furthermore, interconnect driver and receiver are modeled by resistance $R_{d}=100 \Omega$ and loading capacitance $C_{L}=2 f F$. All circuit models are simulated by HSPICE. Below, we present results first for aligned parallel bus lines, and then for the on-chip spiral inductor. For all bus structures, a 1-V step voltage with 10ps rising time is applied to the first line, and all other lines are quiet. The outputs presented in Fig. 7, Fig. 10 and Fig. 11 are measured at the far ends of the last line.

\section{A. Full VPEC Model}

In this part we present the full VPEC model for various structures. We used both FastHenry and formula (27) and (28) to calculate the partial inductance for PEEC model. As shown in the experiment results, the difference of waveforms between the two methods is very small. Therefore we employ the PEEC calculated by formula to obtain the EMRs for VPEC model to efficiently simulate the complicated large systems.

1) Simple aligned parallel bus lines: We consider a fivebit bus, with one-segment per line. Each bus line is $1000 \mu \mathrm{m}$ long, $1 \mu \mathrm{m}$ wide and $1 \mu \mathrm{m}$ thick. The space between lines is $2 \mu \mathrm{m}$. The calculated $L, K, \hat{G}$ and $\hat{R}$ matrices are presented as follows:

$$
\begin{gathered}
L=\left[\begin{array}{lllll}
1.4816 & 1.1820 & 1.0437 & 0.9630 & 0.9059 \\
1.1820 & 1.4816 & 1.1820 & 1.0437 & 0.9630 \\
1.0437 & 1.1820 & 1.4816 & 1.1820 & 1.0437 \\
0.9630 & 1.0437 & 1.1820 & 1.4816 & 1.1820 \\
0.9059 & 0.9630 & 1.0437 & 1.1820 & 1.4816
\end{array}\right] n H \\
K=\left[\begin{array}{lllll}
1.9696 & -1.2091 & -0.1904 & -0.1371 & -0.1749 \\
-1.2091 & 2.6964 & -1.1044 & -0.1231 & -0.1371 \\
-0.1904 & -1.1044 & 2.7052 & -1.1044 & -0.1904 \\
-0.1371 & -0.1231 & -1.1044 & 2.6964 & -1.2091 \\
-0.1749 & -0.1371 & -0.1904 & -1.2091 & 1.9696
\end{array}\right] 10^{9} H^{-1}
\end{gathered}
$$

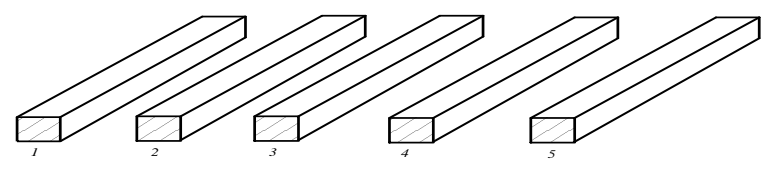

(a) 5-bit bu s

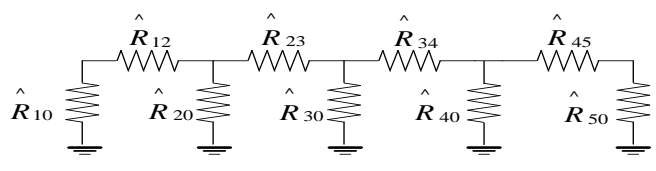

(b) localized VPEC model

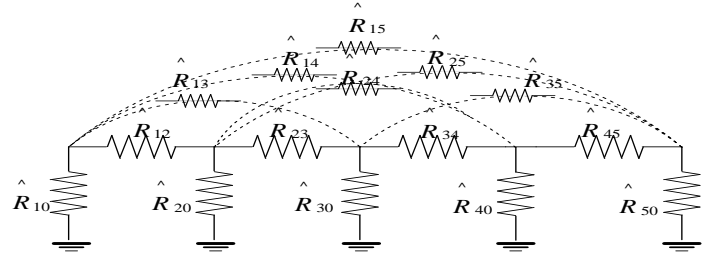

(c) full VPEC model

Fig. 6. The localized and full VPEC models for 5-bit bus lines. For simplicity of presentation, we only show the equivalent magnetic resistance, but not other circuit elements such as resistance, capacitance, and controlled voltage/current source (see Fig. 2).

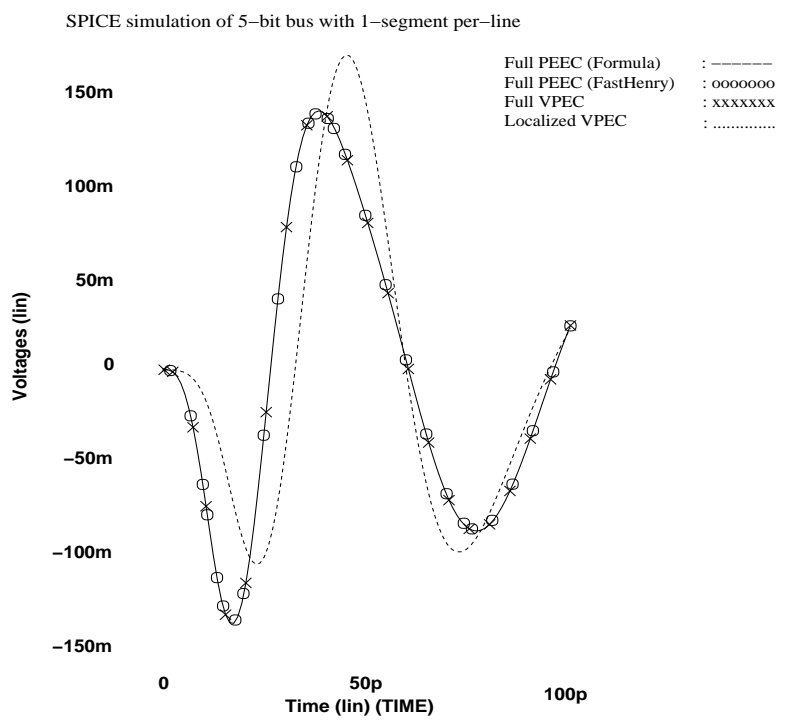

Fig. 7. Waveforms of 5-bit bus with one segment each line.

$$
\begin{aligned}
\hat{G}= & {\left[\begin{array}{lllll}
1.9696 & -1.2091 & -0.1904 & -0.1371 & -0.1749 \\
-1.2091 & 2.6964 & -1.1044 & -0.1231 & -0.1371 \\
-0.1904 & -1.1044 & 2.7052 & -1.1044 & -0.1904 \\
-0.1371 & -0.1231 & -1.1044 & 2.6964 & -1.2091 \\
-0.1749 & -0.1371 & -0.1904 & -1.2091 & 1.9696
\end{array}\right] 10^{3} H^{-1} m^{2} } \\
\hat{R} & =\left[\begin{array}{lllll}
3.8736 & 0.8270 & 5.2533 & 7.2964 & 5.7172 \\
0.8270 & 8.1566 & 0.9054 & 8.1220 & 7.2963 \\
5.2533 & 0.9054 & 8.6494 & 0.9054 & 5.2533 \\
7.2964 & 8.1220 & 0.9054 & 8.1566 & 0.8270 \\
5.7172 & 7.2964 & 5.2533 & 0.8270 & 3.8736
\end{array}\right] 10^{-3} H^{-2}
\end{aligned}
$$

where $\hat{K}$ and $\hat{G}$ matrices differ only by a constant factor $l^{2}$. Similar to the "shielding" effect in the $\hat{K}$ matrix as pointed out in [8], the coupling $\hat{G}_{i j}\left(\hat{R}_{i j}\right)$ between non-adjacent lines is 


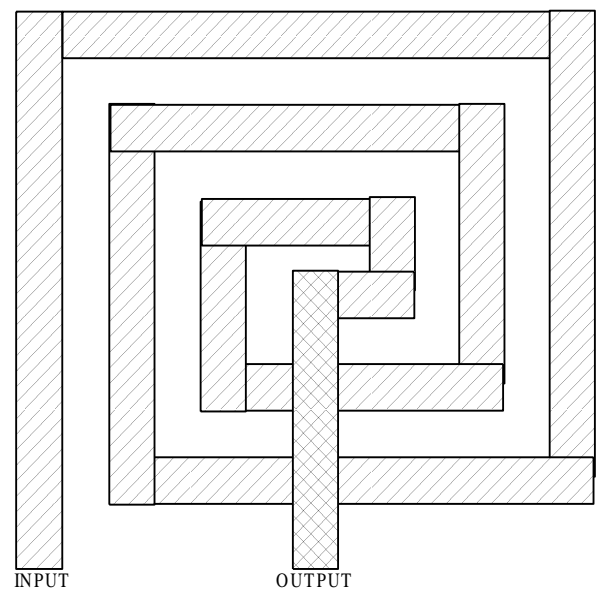

Fig. 8. The geometry of a 3-turn single layer on-chip spiral inductor.

significantly smaller (larger) than that between adjacent lines. For a five-bit bus, we compare in Fig. 6 the circuit of the full VPEC model (with coupling $\hat{R}_{i j}$ between all lines) from this paper, and the circuit of the localized VPEC model (with coupling $\hat{R}_{i j}$ between adjacent lines) from [1]. Clearly as shown in Fig. 7, our full VPEC model and the full PEEC model by FastHenry and formula (27) and (28) obtain identical waveforms, but the localized VPEC model introduces nonnegligible error and is not accurate compared to the full PEEC model.

2) Simulation of non-bus structures: The VPEC model can be employed to simulate monolithic spiral inductors as well as regular aligned parallel bus lines. The on-chip inductors are widely used in CMOS RF circuits, such as LNA and VCO [16], [17]. We have applied VPEC model for the single-layer spiral inductor in Fig. 8. The outer wire length is $1000 \mathrm{um}$, the wire width is $20 u m$, the thickness is $10 u m$, and the spacing between the centers of two adjacent wires is $100 u m$. We assume that wires are segmented as shown in Fig. 8 for both VPEC and PEEC models, apply $1-\mathrm{V}$ voltage at the input port, and measure the response at the output port.

Furthermore, because the h-filaments now have different lengths, the system equation (16) is modified to:

$$
\left(\frac{1}{l_{i}^{2} \hat{R}_{i 0}}+\sum_{j \neq i} \frac{1}{l_{i}^{2} \hat{R}_{i j}}\right) V_{i}+\sum_{j \neq i}\left(-\frac{1}{l_{i} l_{j} \hat{R}_{i j}}\right) V_{j}=\frac{\partial I_{i}}{\partial t}
$$

It directly leads to the following general mapping relation with the $K$ matrix

$$
\hat{R}_{i j}=-\frac{1}{l_{i} l_{j} K_{i j}}, \quad \hat{R}_{i 0}=\frac{1}{l_{i}^{2} K_{i i}+\sum_{j \neq i} l_{i} l_{j} K_{i j}}
$$

The simulation result is presented in Fig. 9. The PEEC models by FastHenry and formula (27) and (28) generate identical waveforms, and the VPEC model can reproduce the result of the PEEC model for the non-bus line structures as well. Therefore the VPEC model is suitable to be applied for the general layout. Note that when the number of the wires of the simulated system is small, there is no eminent simulation speedup observed. However, in the following experiments with larger wire segments, we found the simulation effort in the full

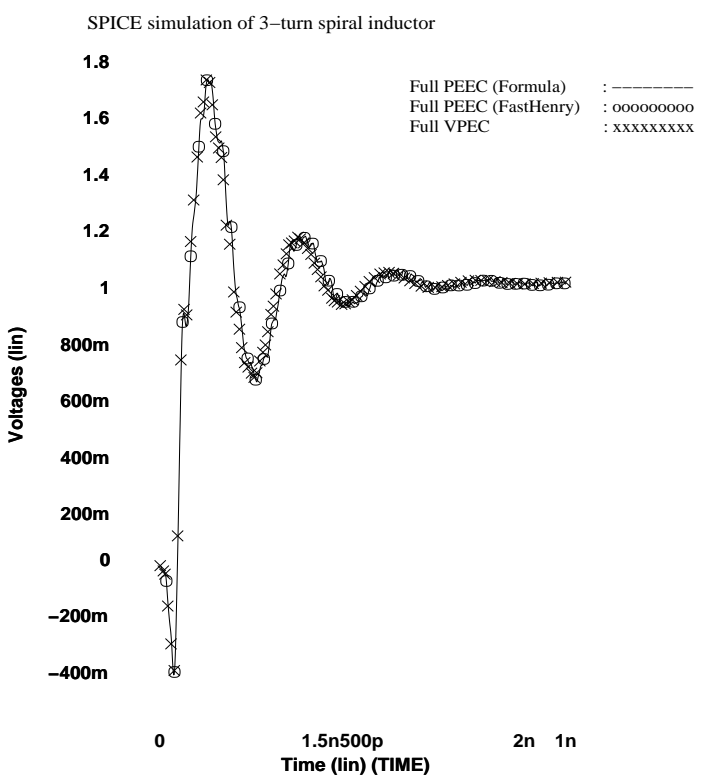

Fig. 9. Waveforms of the 3-turn single layer on-chip spiral inductor.

VPEC model is much less than the full PEEC model, even without the sparsification.

\section{B. Sparsification for VPEC Model}

We study two sparsification procedures, numerical sparsification and 2D geometry based sparsification for various bus line structures in this part.

1) Numerical Sparsification: As explained in Section 3.2, $\hat{G}$ matrix is passive and diagonal dominant. Therefore, smallvalued off-diagonal elements can be truncated without loss of passivity. For example, setting the truncating threshold as 0.09 where any off-diagonal element smaller than 0.09 of its correspondent diagonal element is truncated results in the following truncated $\hat{G}$. (denoted as truncated VPEC in Table I) for the above five-bit bus:

$$
[\mathrm{G}]=\left[\begin{array}{lllll}
1.9696 & -1.2091 & -0.1904 & 0 & 0 \\
-1.2091 & 2.6964 & -1.1044 & 0 & 0 \\
0 & -1.1044 & 2.7052 & -1.1044 & 0 \\
0 & 0 & -1.1044 & 2.6964 & -1.2091 \\
0 & 0 & -0.1904 & -1.2091 & 1.9696
\end{array}\right] 10^{3} H^{-1} m^{2}
$$

Applying (17) to truncated $\hat{G}$ leads to the following truncated $\hat{R}$ :

$$
[\hat{R}]=\left[\begin{array}{lllll}
3.8736 & 0.8270 & 5.2533 & \infty & \infty \\
0.8270 & 8.1566 & 0.9054 & \infty & \infty \\
\infty & 0.9054 & 8.6494 & 0.9054 & \infty \\
\infty & \infty & 0.9054 & 8.1566 & 0.8270 \\
\infty & \infty & 5.2533 & 0.8270 & 3.8736
\end{array}\right] 10^{-3} \mathrm{H}^{-2}
$$

Fig. 10 plots the simulation results under our numerical sparsification for a 128-bit bus with one segment per line, where the sparse factor is the ratio between the numbers of circuit elements in the truncated and full VPEC models. The waveform difference is small in terms of the noise peak for sparse factors up to $30.5 \%$. Table I summarizes the truncation setting and simulation result, where the values in parentheses of column 1 are truncating thresholds, and the runtime includes both SPICE simulation and matrix inversion in case of VPEC models. The average voltage differences, and associated standard deviations are calculated for all time steps in SPICE simulation. One can see from the table that up to $30 \mathrm{X}$ 


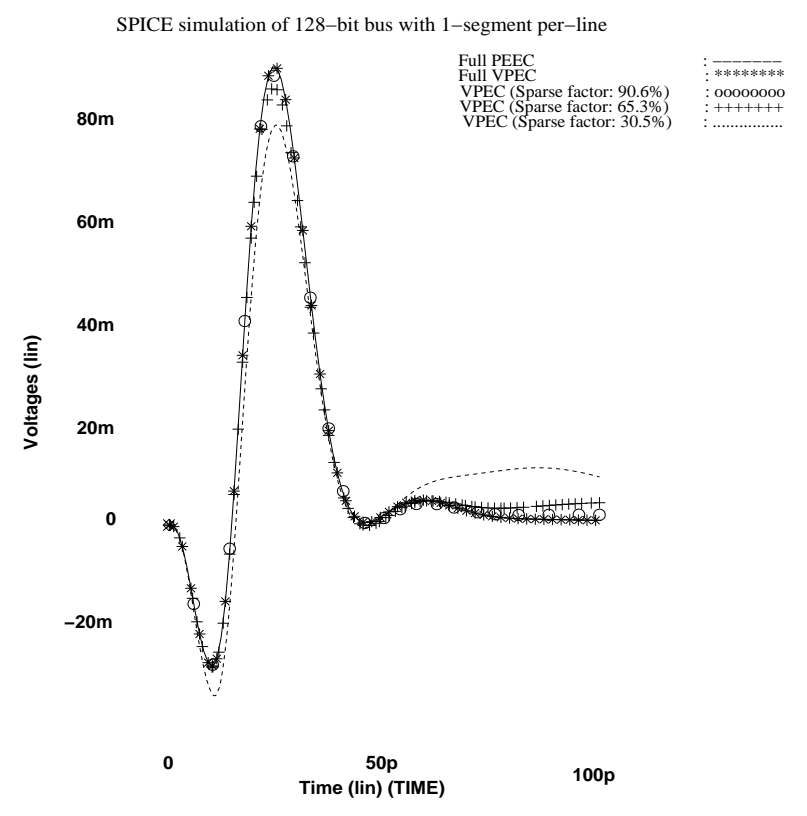

Fig. 10. Waveforms in numerical sparsification of VPEC model for 128-bit bus with one segment each line.

speedup is achieved when the average waveform differences is up to $0.377 \mathrm{mV}$, less than $1 \%$ of the noise peak. A much bigger speedup factor can be expected as a much higher waveform difference can be tolerated in practice. Compared to the full PEEC model, the full VPEC simulation is 7X faster, due to the fact that the VPEC model has a bit more resistances and coupled current/voltage sources but much fewer inductances. The negligible difference between the full VPEC and PEEC simulations is due to the numerical matrix inversion.

2) Geometry Based Sparsification: We study the geometry based sparsification for segmented buses by defining a truncating window $\left(N_{W}, N_{L}\right)$, where $N_{W}$ and $N_{L}$ are the numbers of coupled segments in directions of wire width and length, respectively. We further define the couplings along wire length as the forward coupling same as in [18], and along wire width as the aligned coupling. For each wire segment, the circuit model only contains $\hat{R}_{i j}$ within the truncating window of the segment, and is called windowed VPEC model in Table II. We consider a 32-bit bus with eight segments per line and four different windows: $(32,8),(32,2),(16,2)$ and $(8,2)$. Furthermore, we apply the normalized model [19] to VPEC (called normalized VPEC model in Table II) for the bus lines with $n$ segments per line. If the EMR between any two bus lines without segmentation is $\hat{R}_{i j}$, the EMR for each pair of aligned segments is $\hat{R}_{i j} \cdot n^{2}$, and is zero for non-aligned segments.

We plot simulations under different models in Fig. 11, and summarize the experiment setting and result in Table II. There is a smooth trade-off between runtime and accuracy for different window sizes. We first compare results of different truncating windows. The window $(8,2)$ achieves the highest speedup of $30 \mathrm{X}$ and the largest difference of about $0.2 \mathrm{mV}$ on average, less than $2 \%$ of the noise peak, and the window $(32,2)$ has the highest accuracy with $0.06 \mathrm{mV}$ on average but a reduced speedup of 10X. Furthermore, we compare

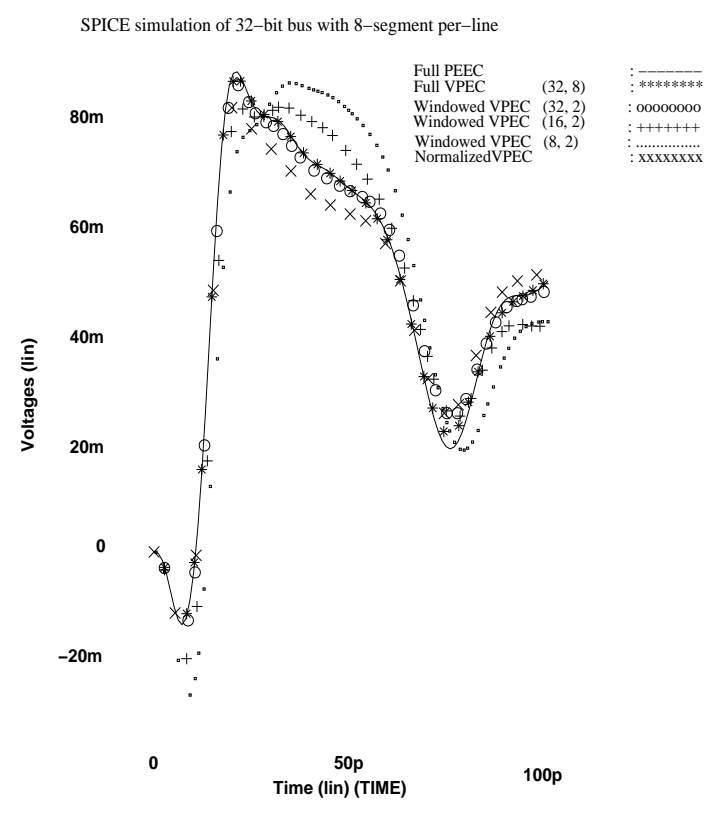

Fig. 11. Waveforms in geometry based sparsification of VPEC model for 32-bit bus with 8 segments each line.

the normalized model to the window $(16,2)$ with a similar complexity. The windowing technique is faster but has a larger standard deviation. The normalized model implicitly considers the forward coupling between all non-aligned segments, and the window $(16,2)$ considers forward coupling between adjacent segments only. The small difference between the two models implies that the forward couplings between nonadjacent segments may be negligible, which is also indicated by the small difference between windows $(32,8)$ and $(32,2)$. However an $N_{W}$ much larger than $N_{L}$ (as shown in Table II) is needed to archive a high accuracy. This implies that the aligned coupling is stronger than the forward coupling.

\section{Runtime Scaling}

We compare runtimes to analyze parallel bus structures using the full PEEC model, full VPEC model, and windowed VPEC model, respectively. The runtime for the full or windowed VPEC model includes both SPICE simulation and matrix inversion. We first consider one segment per line, and plot runtimes in Fig. 12 (a). The full PEEC and VPEC models can only handle the bus with up to 256 bit because SPICE can not further allocate enough memory. But the windowed VPEC model can handle the bus with up to 1024 bit. For the 256-bit bus, the full and windowed VPEC model is 47X (185.39s vs. 8726.85s) and over 1000X faster than the full PEEC model, respectively. Our experiment assumed a truncating window of $(8,1)$ because $N_{W}=8$ for a truncating window $\left(N_{W}, N_{L}\right)$ has a reasonably good error bound as shown in Table II. It is easy to see that the windowed VPEC has a slow runtime scaling with respect to the increase of the bus width.

We further conducted the experiment considering the distributed interconnect model with eight segments each line, and plot the runtimes in Fig. 12 (b). Based on Table II, we assume a $(8,2)$ truncating window for the windowed VPEC model. 
The full PEEC and VPEC models can only handle 32-bit bus, but the window VPEC is able to handle the 128-bit bus at a much reduced runtime. The scaling of the runtime using the windowed VPEC model is super-linear, but its increase is much slower than that for the full PEEC and VPEC models.

In both experiments, the full VPEC model achieves identical waveform and the windowed VPEC model has a very small waveform difference when compared to the full PEEC model. The observation is similar to Fig. 11.

\section{CONCLUSIONS AND Discussions}

The primary contribution of this paper is to derive from first principles both integration and inversion based VPEC models for multiple inductive interconnects. Using equivalent resistance network and controlled voltage and current sources to replace inductance network, the full VPEC model is as accurate as the full PEEC model but takes less simulation time. We have observed a speedup of $47 \mathrm{X}$ for simulating 256 wire segments in a bus structure. Further, the resulting circuit element matrix $\hat{G}$ in the VPEC model is passive and strictly diagonal dominant. This leads to easy sparsification methods with guaranteed passivity. We have presented both numerical and geometry based sparsification methods. When compared to the full PEEC and VPEC models, the sparsified VPEC models achieve orders of magnitude speedup in circuit simulation and produce waveforms with very small error.

Because matrix $\hat{G}$ and matrix $K$ from [7] differ only by constant factors, our study can be used to justify from first principles the $K$ matrix based sparsification methods. One can use geometry integration to calculate the matrices $\hat{R}$ and $\hat{G}$. Therefore, the matrix $K$ can be calculated directly by geometry integration without inverting the partial inductance matrix. Note that SPICE is able to directly simulate VPEC model but not $K$-matrix based model.

In essence, our VPEC sparsification can be viewed as full VPEC generation followed by sparsification. Although we have shown that the locality of VPEC illustrated in [1] does not hold in general, the method in [1] can be viewed as a special case with the smallest truncating window size for the direct generation of a sparsified VPEC model. The direct method with bounded accuracy loss is attractive if it is more efficient compared to the inversion of the full inductance matrix as such inversion is needed by the sparsification methods in this paper. Efficient methods for direct sparsification with bounded accuracy loss are planned as our future work on VPEC model.

\section{REFERENCES}

[1] A. Pacelli, "A local circuit topology for inductive parasitics," in ICCAD, pp. 208-214, 2002.

[2] J. Lillis, C. Cheng, S. Lin, and N. Chang, High-performance interconnect analysis and synthesis. John Wiley, 1999.

[3] A. E. Ruehli, "Equivalent circuits models for three dimensional multiconductor systems," IEEE Trans. on MIT, pp. 216-220, 1974.

[4] Z. He, M. Celik, and L. Pillegi, "SPIE: Sparse partial inductance extraction," in 34th DAC, pp. 137-140, 1997.

[5] K. Shepard and Z. Tian, "Return-limited inductances: A practical approach to on-chip inductance extraction," IEEE Trans. on CAD, vol. 19, no. 4, pp. 425-436, 2000.

[6] B. Krauter and L. Pileggi, "Generating sparse partial inductance matrices with guaranteed stability," in ICCAD, pp. 45-52, 1995.
[7] A. Devgan, H. Ji, and W. Dai, "How to efficiently capture on-chip inductance effects: introducing a new circuit element $\mathrm{K}$," in ICCAD, pp. $150-155,2000$.

[8] H. Ji, A. Devgan, and W. Dai, "Ksim: A stable and efficient RKC simulator for capturing on-chip inductance effect," in 38th DAC, pp. 379-384, 2001.

[9] M. Beattie and L. Pileggi, "Efficient inductance extraction via windowing," in DATE, pp. 430-436, 2001.

[10] G. Zhong, C. Koh, and K. Roy, "On-chip interconnect modeling by wire duplication," in ICCAD, pp. 341-346, 2002.

[11] M. Kamon, M. Tsuk, and J. White, "FastHenry: a multipole-accelerated 3D inductance extraction program," IEEE Trans. on MIT, 1994.

[12] K. Narbos and J. White, "FastCap: A multipole accelerated 3D capacitance extraction program," IEEE Trans. on CAD, vol. 10, no. 11, pp. 1447-1459, 1991.

[13] S. Gersgorin, "Uber die abgrenzung der eigenverte einer matrix," in Izv. Akad. Nauk., USSR, no. 7, pp. 749-754, 1931.

[14] F. Grover, "Inductance calculations: Working formulas and tables," in Dover Publications, New York, 1946.

[15] X. Qi, G. Wang, Z. Yu, R. Dutton, T. Young, and N. Chang, "Onchip inductance modeling and RLC extraction of VLSI interconnects for circuit simulation," in CICC, pp. 487-490, 2000.

[16] J. J. Zhou and D. Allstot, "Monolithic transfomers and their application in a differential CMOS RF low-noise amplifier," IEEE Journal of SoildState Circuits, vol. 33, no. 12, pp. 2020-2027, 1998.

[17] J. Craninckx and M. Steyaert, "A 1.8-GHz low-phase-noise CMOS VCO using optimized hollow spiral inductors," IEEE Journal of Soild-State Circuits, vol. 32, no. 5, pp. 736-744, 1997.

[18] T. Lin, M. W. Beattie, and L. T. Pileggi, "On the efficacy of simplified 2D on-chip inductance models," in 39th DAC, 2002.

[19] M. Xu and L. He, "An efficient model for frequency-based on-chip inductance," in IEEE/ACM International Great Lakes Symposium on VLSI, 2001. 


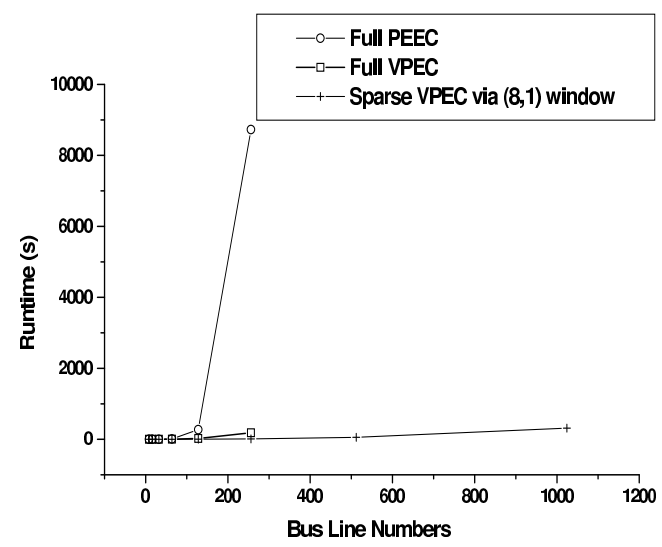

(a)

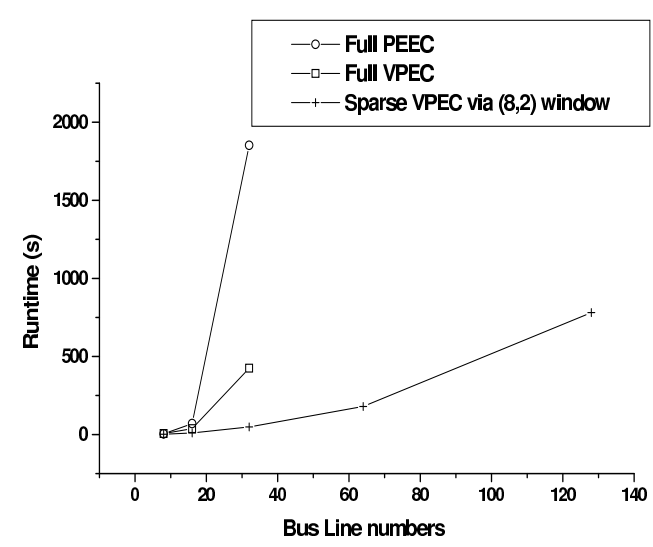

(b)

Fig. 12. Runtime to simulate multiple bus lines using the full PEEC, full VPEC, and sparsified VPEC models: (a) multiple bus lines with one segment each line; (b) multiple bus lines with eight segments each line.

\begin{tabular}{|l|l|l|l|l|}
\hline $\begin{array}{l}\text { Models and } \\
\text { Truncating Thresholds }\end{array}$ & $\begin{array}{l}\text { No. of } \\
\text { Elements }\end{array}$ & $\begin{array}{l}\text { Run-Time } \\
\text { and Speedup }\end{array}$ & $\begin{array}{l}\text { Avg. Volt. } \\
\text { Difference }\end{array}$ & $\begin{array}{l}\text { Standard } \\
\text { Deviation }\end{array}$ \\
\hline Full PEEC & 8256 & $281.02 s(1 \mathrm{X})$ & $0 \mathrm{~V}$ & $0 \mathrm{~V}$ \\
\hline Full VPEC $(0.0)$ & 8256 & $36.40 s(7 \mathrm{X})$ & $-1.64 \mathrm{e}-6$ & $3.41 \mathrm{e}-4 \mathrm{~V}$ \\
\hline Truncated VPEC $(5 \mathrm{e}-5)$ & 7482 & $30.89 s(9 \mathrm{X})$ & $4.64 \mathrm{e}-6 \mathrm{~V}$ & $4.97 \mathrm{e}-4 \mathrm{~V}$ \\
\hline Truncated VPEC $(1 \mathrm{e}-4)$ & 5392 & $19.55 s(14 \mathrm{X})$ & $1.29 \mathrm{e}-5 \mathrm{~V}$ & $1.37 \mathrm{e}-3 \mathrm{~V}$ \\
\hline Truncated VPEC $(5 \mathrm{e}-4)$ & 2517 & $8.35 s(28 \mathrm{X})$ & $3.77 \mathrm{e}-4 \mathrm{~V}$ & $5.20 \mathrm{e}-3 \mathrm{~V}$ \\
\hline
\end{tabular}

TABLE I

RESULTS OF NUMERICAL SPARSIFICATION

\begin{tabular}{|l|l|l|l|l|}
\hline $\begin{array}{l}\text { Models and } \\
\text { Window Sizings }\end{array}$ & $\begin{array}{l}\text { No. of } \\
\text { Elements }\end{array}$ & $\begin{array}{l}\text { Run-Time } \\
\text { and Speedup }\end{array}$ & $\begin{array}{l}\text { Avg. Volt. } \\
\text { Difference }\end{array}$ & $\begin{array}{l}\text { Standard } \\
\text { Deviation }\end{array}$ \\
\hline Full PEEC & 32896 & $2535.48 s(1 \mathrm{X})$ & $0 \mathrm{~V}$ & $0 \mathrm{~V}$ \\
\hline Full VPEC(32,8) & 32896 & $772.89 s(3 \mathrm{X})$ & $1.00 \mathrm{e}-5 \mathrm{~V}$ & $6.26 \mathrm{e}-4 \mathrm{~V}$ \\
\hline Windowed VPEC $(32,2)$ & 11392 & $311.22 s(8 \mathrm{X})$ & $5.97 \mathrm{e}-5 \mathrm{~V}$ & $1.84 \mathrm{e}-3 \mathrm{~V}$ \\
\hline Windowed VPEC $(16,2)$ & 3488 & $152.57 s(16 \mathrm{X})$ & $-1.23 \mathrm{e}-4 \mathrm{~V}$ & $4.56 \mathrm{e}-3 \mathrm{~V}$ \\
\hline Windowed VPEC $(8,2)$ & 2240 & $85.14 s(32 \mathrm{X})$ & $-2.17 \mathrm{e}-4 \mathrm{~V}$ & $8.91 \mathrm{e}-3 \mathrm{~V}$ \\
\hline Normalized VPEC & 4224 & $255.36 s(10 \mathrm{X})$ & $-6.05 \mathrm{e}-4 \mathrm{~V}$ & $2.96 \mathrm{e}-3 \mathrm{~V}$ \\
\hline
\end{tabular}

TABLE II

RESULTS OF GEOMETRY BASED SPARSIFICATION 